\title{
Optimization Production Planning Using Fuzzy Goal Programming Techniques
}

\author{
Houshang Taghizadeh ${ }^{1}$, Ardeshir Bazrkar ${ }^{2,3}$ \& Mohammad Abedzadeh ${ }^{2,3}$ \\ ${ }^{1}$ Department of Management, Tabriz Branch, Islamic Azad University, Tabriz, Iran \\ ${ }^{2,3}$ Department of Industrial Management, Tabriz Branch, Islamic Azad University, Tabriz, Iran \\ ${ }^{2,3}$ Department of Industrial Management, East Azerbaijan Science and Research Branch, Islamic Azad \\ University, Tabriz, Iran. \\ Correspondence: Houshang Taghizadeh, Department of Management, Tabriz Branch, Islamic Azad University, \\ Tabriz, Iran. E-mail: taghizadeh@iaut.ac.ir
}

Received: March 10, 2015

Accepted: March 25, 2015

Online Published: July 10, 2015

doi:10.5539/mas.v9n9p68

URL: http://dx.doi.org/mas.v9n9p68

\begin{abstract}
In the past two decades, ideal planning has been used for the multiple criteria decision making problem solving. The issue is raised here is that how to do a program to achieve the best and most comprehensive program that it has well run ability? Because of costly medical equipment production process, it should pay more attention to production planning. In this research, this was carried out in the company's beta; we are looking to fulfill two objectives, reducing the cost of production, increase revenue leading to increased profits. For the realization of these goals, we use the method of fuzzy planning, according to studies, we use simple collective models and collective weighted method. According to the existing information and problem variables and TIVARY model, we find that the model leads to production costs and increasing income. According to the results of the research, we realized that for the realization of the Beta purposes, we should use TIVARY collective method. This study has expanded the discussion of optimization production planning using fuzzy goal programming techniques. Goal programming was used in various issues such as decision making. The most important goal programming restrictions is unclear goals. Fuzzy goal programming provided, it investigate fuzzy purpose in unknown level.
\end{abstract}

Keywords: production planning, goal programming, Fuzzy Logic, TIVARY model

\section{Introduction}

With the extensive industrial production process and need to meet the market demand with the highest quality and quantity, many problems appear. At the present time, the obstacles are not technical problems, more relevant to maximize the use of minimal resources. These references will be converted to usable products and quality, if used correctly. If it is not used correctly, not only do not create added value but cause wasting resources and increasing losses in production. In the matters, accurate identification of the needs of the market and to align with it in order to produce final products with the maximum value is very important. To achieve this goal, you should be paying attention to a few basic tips. First, The amount of required by any of the existing resources, including raw materials and machinery and manpower to be determined for each product unit, So that we can more accurately defining the production program. Then the available resources should be to estimate in the desired timeframe. Finally, the program is set to be the most productive efficiency and effectiveness in production and supply demand (Anvarirostami \& Tabatabai, 1998). Production planning follows amount of production capacity and amount of product demand; it includes mid-term customers' orders between 3 to 18 months. The main goals of production planning are as follows:

1. Coordination between production rates with the amount of order. 2. Decisions regarding hiring, dismissal, overtime, postponing orders, optimal inventory levels, side agreements, determine the proper resources for the production (Aouni \& kettani, 1997). The medical supplies industry complies with Building system based on order, because of the high cost of production and specifying consumption process. Due to nature of demand, production planning in this industry has a lot of important, in a way that it will have high flexibility and be able to coordinate with demands. Because of the high cost of producing medical supplies, we should pay more attention to production planning. So we should be looking for a way to reach the highest level of performance in accurately and 
scientifically and high-functionality method. Operations research is science which gives us the possibility to do using linear programming method. We can use several methods of operations research for production planning. So we have to look for a suitable manner with the help of operations research, using it leads to best production planning with considering the existing limitations, and complexity of the production process. Practical method in this research is fuzzy goal programming. This method can consider opinions of managers and decision makers in the industry. Decision makers imagine desirable results for purposes, fuzzy goal programming model convert this desirable levels to mathematical language. Its results have most coordination with decision makers' optimal. It is worth nothing that goal programming model can consider two types of soft and hard constraints. The difference between these two types of restrictions is that in fact, we can go beyond the limits. Deviation from the values of the right will cause the soft limits. There are limits that any diversion of them is not possible they are called hard constraints (Chen, 2001).

According to the basic objectives of the research, the following research questions are expressed:

1.1 Does the application of fuzzy programming model increase production revenue?

1.2 Does the running fuzzy goal programming can reduce cost?

\section{Research Literature}

Production planning is one of the most important branches of industry-related applications which are obtained from the combination of the various techniques of industrial engineering with mathematical optimization techniques and heuristics.

Large branches in engineering industries haven't high capacity of reception a variety of math and conceptual models. According to the definition, production planning means decisions about the resources that the organization needs for the future and allocation of these resources will be possible for the number of needed product and the lowest cost. In fact, production planning is meant to create a range for future production in organization. According to above, two main goals will be considered for production planning:

-Determining production plans based on cost and management policies regarding customer service issues, financial and sustainability task. With this program, we can decide where we need to add capacity.

-Contribute to the management in order to show the impact of various policies on costs, the amount of inventory and production (Chase and Tsai, 2011).

\subsection{Goal Planning}

In Multi Criteria Decision Making methods, we use several measures instead of using a measure of optimality. Multi-criteria decision making models can be divided into for multi-objective and multi-criteria decision-making models. In general, multi-objective models are used to design and multi-criteria models are used to select the top option. The main difference between the multi-objective decision making and multi-criteria models is that the former is defined on continuous decision space and the latter is defined on the discrete decisions space. Goal programming, first by Charnes and Cooper and Ferguson was introduced in 1955 (Charnes et al., 1995) but in 1961, the goal programming model is released by Charnes and Cooper (Charnes and Cooper, 1961). Methods of goal programming have common context. Their aim is to minimize the unfavorable deviations from purposes (Agawal \& Kholi, 1991). Goal programming is linear programming which can be used for multiple and conflicting goals. In this case, the model must be solved in such a way that each of the objectives to be achieved. Therefore, the sum of the deviations from the ideal should be minimized in objective function.

It is important that measure deviations from the ideal should have a single scale, because deviations with different scales cannot be collected. At First, you should do unsealed all deviations using one of methods unsealed, they must have a common unit, and so all deviations are collected on the target function (Chase, 2001). The General form of the programming model is as follows:

In this model:

In this model:

$$
\begin{aligned}
& \operatorname{Min} A=\left\{g_{1}\left(n_{1}, p_{1}\right), g_{2}\left(n_{2}, p_{2}\right), \ldots, g_{k}\left(n_{k}, p_{k}\right)\right\} \\
& \text { S u b ject to : } \\
& f_{i}(x)+n_{i}-p_{i}=b_{i} \quad i=1,2, \ldots, m \\
& \quad \mathrm{x}, \mathrm{n}_{\mathrm{i}}, \mathrm{p}_{\mathrm{i}} \geq 0
\end{aligned}
$$

$\mathrm{A}$ is target function 
$\mathrm{g}_{\mathrm{k}}\left(\mathrm{n}_{\mathrm{k}}, \mathrm{p}_{\mathrm{k}}\right)$ Is perverted variables function in $k$

$\mathrm{b}_{\mathrm{i}}$ Is the right amount of aspiration $i$

$\mathrm{n}_{\mathrm{i}}$ Is negative deviation from ideal $i$

$\mathrm{p}_{\mathrm{i}}$ Is positive deviation from the ideal $i$

$\mathrm{f}_{\mathrm{i}}(\mathrm{x})$ Is the objective function of the decision variables $i$ (Agawal \& Kholi, 1991)

In usual ideal planning models to achieve an acceptable solution, total undesirable deviations looks to be at least. Undesirable deviations include positive or negative deviations or both.

\subsection{Fuzzy Set}

Fuzzy set shows a group of members, in this set, there is no clear boundary between the members and non-members. $\mathrm{x}$ elements are members of $\mathrm{X}$ set. Therefore, fuzzy set $\mathrm{C}$ from $\mathrm{X}$ defines as set of couples.

$$
\mathrm{C}=\left[\mathrm{X}, \mu_{\mathrm{i}}(\mathrm{X})\right] \quad \mathrm{X} \in \mathrm{X}
$$

Where, the membership function is membership grade X in fuzzy set C. Membership function is between [0-1], so that, 0 value shows lowest grade of membership and 1 value is the highest grade of membership.

\subsubsection{Memberships Function of Crisp Sets:}

Membership function is defined as follows:

$$
\mu_{A}(x)= \begin{cases}1 & x \in A \\ 0 & x \notin A\end{cases}
$$

It can be expressed so that, if $\mathrm{x}$ is belonging to set $\mathrm{A}$, the membership function value is one, otherwise the value will be zero. It can be observed that, the domain of the membership function is reference set and its range is set of two members of $\{0,1\}$.

For example, suppose that in reference set $X=\{1,2,3,4,5\}, A=\{1,3\}$ is a subset of $X$.

The set A can be indicated as follows:

$$
\mu_{A}= \begin{cases}1, & x=1,3 \\ 0, & x=2,4,5\end{cases}
$$

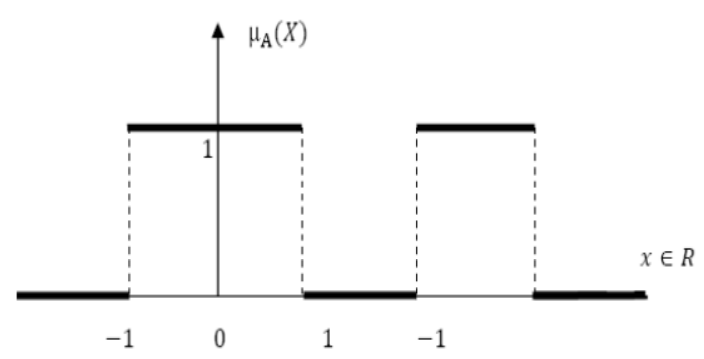

Figure 1. Membership function of a crisp set

\subsubsection{Membership Function of Fuzzy Sets}

As it has explained, membership function of crisp sets is a set of two members $(0,1)$ that can be shown as follows: 


$$
\mu_{A}: x \rightarrow\{0,1\}
$$

So, if the range of function is two member function, it is called a fuzzy set of X. in function $\mu_{\mathrm{A}}(\mathrm{x})$, the nearer value to one shows, more depending of $\mathrm{x}$ to the set $\mathrm{A}$ and the nearer value to zero shows, less depending of $\mathrm{x}$ to the set A.

Accordingly, if $\mu_{\mathrm{A}}(\mathrm{x})=1$, then $\mathrm{x}$ is located in $\mathrm{A}$, and if $\mu_{\mathrm{A}}(\mathrm{x})=0, \mathrm{x}$ is not member of $\mathrm{A}$. So, fuzzy sets and their membership functions are generalization of crisp functions (Akoz and Petrovic, 2007).

Membership functions of fuzzy sets are shown as follows:

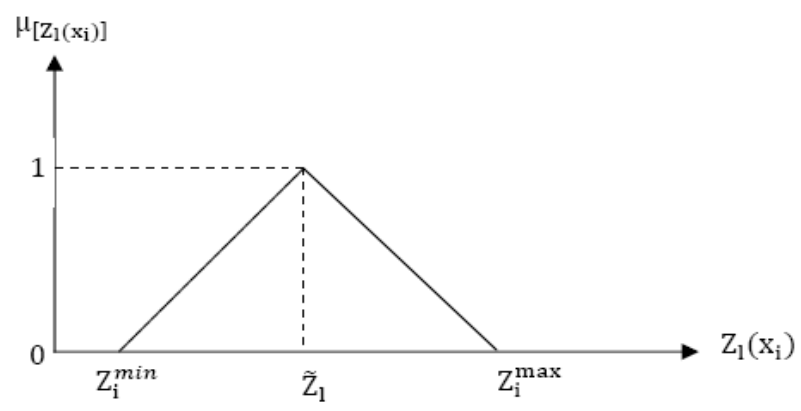

Figure 2. Membership function of a fuzzy set

\subsection{Fuzzy Goal Programming}

After Narsyman introduced fuzzy goal programming model, the studies were carried out on a fuzzy goal programming models to enhance computing performance. Hanan entered diversion goal programming variables into fuzzy goal programming model. To solve the model, just an ordinary linear programming formulation was necessary; Of course, this leads to an increase of the variables in formulating model (Chen and Tsai, 2001). Priority of objectives in the planning of an ideal planning is an important issue. For some causes are more important than others. Access to them is more important than others. Two different methods have been proposed for consideration of the importance.

\subsubsection{Sequential Method}

Decision-maker may consider priorities for goals, and objectives, assign different importance, which is known as sequential method. Solution is that objectives are divided into $\mathrm{K}$ priority. $\mathrm{K}$ is the number of fuzzy goals minus one. Then, $\mathrm{k}$ subsidiary issue is resolved. The achievement of the above objectives will be added to the least important issues as constraints (Chernes et al., 1995).

The achievement of each of the sub-problems can be achieved. Although this method solves the model taking into account the importance of arbitrary decisions, efficiency is greatly reduced. In hierarchical structure which is provided by TIVARY, like other sequential methods, top objectives must be achieved. The higher objectives should be more important than the lower goals. Wang and Fu are used different membership functions to express the objectives priority which were obtained by using fuzzy set operations such as expansion and contraction. Chen and Tsai model suggests model that priorities objectives according to importance expressed by decision-maker. In this way, the decision maker determines the minimum values for each level of achievement. Objectives are modeled using appropriate access levels. Akoz and Petrovic, raised a fuzzy goal programming model where the importance level of aspiration, precisely defined and phase relationships are shown. This model proposed a new structure for objective function. The objective function is the sum of all achieving levels to goals and degree of satisfaction. In fact, the precise determination of the priority objectives in multi-objective problems is not easy. Uncertainty is unavoidable in expressing significance of goals. Or prioritization of objectives, from perspective of decision maker is ambiguous. The decisions and dependencies space between goals is another factor that influences on determination of objectives. Therefore, we need a fuzzy goal programming model to the uncertainty considered and provide a flexible tool for decision (Akos and Petrovic, 2007).

\subsubsection{Gravimetric Method}

In more precise phrase, fuzzy weights show that the optimal value of an ideal, to determine the significance of goals than each other. Of course, this approach produces unacceptable results. Hanan and Dubbing used different 
weights to illustrate the importance of goals. They enter ideal weight to model as objective function coefficients. Hanan used weights to demonstrate the importance of goals. He assigns different weights to the objectives. In objective function, the sum of the weighted deviations from the center of the triangular membership functions is minimized. This method makes use of isosceles triangular membership functions (Anvarirostami and Tabatabai, 1998).

\section{Research Methodology}

Research methodology is applicable. To collect the data, we use the method prescriptive field models. Prescriptive models generally use a scientific approach to problem solving. The result of interaction and variables relationship are used to decision makers. Problem-solving approach can be summarized in 6 steps:

1. Observation 2. Definition of the problem. 3. Making model. 4. Resolving model 5.Validation. 6. Implementation. In this paper, after reviewing the Beta Company's production process; the use of facilities and resources to produce each unit of product in the specified time interval is obtained. After this step, the levels of resources were determined to establish the available limits to justify the space obtained for the final answer. After ensuring the accuracy of the information provided, we developed a fuzzy goal programming model. The intended targets of decision makers identify and weighed. The limitations of the model were taken as definitive. The next step was to collect the data required as input to models such as the ingredients of each product, revenue which were collected using interview techniques, observation and company database.

It is worth noting that because the fuzzy goal programming, optimal levels of each of the goals must be specified and allowed each interval, specified by decision makers, Comments are used to obtain the desired range.

\section{Analysis of Research data}

The study was discussed on production optimization using fuzzy goal programming. The validity of each study is applicability. Also, different models were examined to analyze the cost and increase profits. Model implemented in the company's functional beta. In analyzing the data, production planning key variables was identified using interview techniques.

\subsection{Research Variables}

Variables are divided into two groups: deterministic and non-deterministic.

\subsubsection{Deterministic Variables}

These variables were included in both groups. The first group includes products that are indicated by the letter $\mathrm{y}$. The second group consists of raw materials, which are indicated by the letter $\mathrm{X}$.

\section{$Y 1, Y 2$ and $Y 3$}

\section{$X 1 X 2$ and $X 3$}

\subsubsection{Non-Deterministic Variables (Fuzzy)}

1.2.1.4 The total cost of production $\left(b_{1}\right)$ : Managers often decide to reduce their prices as much as possible; however, to achieve the lowest cost may not be exact. Then, we use fuzzy criteria for total costs.

2.2.1.4 Total sales revenue $\left(b_{2}\right)$ : Managers often decide to increase their revenue as much as possible; however, to achieve the highest revenue may not be exact. Then, we use fuzzy criteria for total revenue.

\subsection{Multi-Objective Problem Definition}

Due to the variables defined in the previous section and the information in our database, we can explain the following multi variable. The number of product y1 is $50 \% \mathrm{X} 1,25 \% \mathrm{X} 2$ and $25 \% \mathrm{X} 3.16$ number of this product can be produced maximum. Thus, the constraints associated with this product $\left(Y_{1}\right)$ include:

$$
\begin{aligned}
& 0.5 x_{1}+0.25 x_{2}+0.25 x_{3} \geq y_{1} \\
& y_{1} \leq 16
\end{aligned}
$$

Each product $Y 2$ is a combination of $15 \% \mathrm{X} 2,25 \% \mathrm{X} 1$ and $60 \% \mathrm{X} 3.101$ number of this product can be produced

$$
\begin{aligned}
& 0.25 x_{1}+0.15 x_{2}+0.60 x_{3}=y_{2} \\
& y_{2} \leq 101
\end{aligned}
$$

maximum. Thus, the onstraints associated with this product include: 
Each product $\mathrm{Y} 3$ is a combination of $60 \% \mathrm{X} 1$ and $40 \% \mathrm{X} 2.390$ number of this product can be produced maximum. Thus, the constraints associated with this product include:

$$
\begin{aligned}
& 0.60 x_{1}+0.40 x_{2}=y_{3} \\
& y_{3} \leq 390
\end{aligned}
$$

Revenue from product sales in Beta is as follows:

$$
11627 x_{1}+6288 x_{2}+7107 x_{3}
$$

Total revenue of sales is 3658894 that it used as b2 criteria.

According to this description, the issue of several linear programming is as follows:

$$
\begin{aligned}
& \text { Max } 16500 y_{1}+22604 y_{2}+2851 y_{3} \\
& \text { Min } 1162 x_{1}+628 x_{2}+710 x_{3} \\
& \text { S.t } \\
& 0.5 x_{1}+0.25 x_{2}+0.25 x_{3}=y_{1} \\
& 0.25 x_{1}+0.15 x_{2}+0.60 x_{3}=y_{2} \\
& 0.60 x_{1}+0.40 x_{2}=y_{3} \\
& y_{1} \leq 16 \\
& y_{2} \leq 101 \\
& y_{3} \leq 390 \\
& x_{1}, x_{2}, x_{3}, y_{1}, y_{2}, y_{3} \geq 0
\end{aligned}
$$

Above problem is a multi-objective problem that it can be solved by the techniques of multi objective problems. One of these methods is goal programming method. As we explained in the previous section, Most of the information we receive from the environment aren't accurate. Because of its importance for decision making for purposes, we consider them as fuzzy. In fact, the purpose of this section is the implementation of a fuzzy goal programming model to reducing costs and increasing profits. In this first part, we consider dubbing simple additive model. Then we use the weighted additive model for the implementation of goal programming model.

\subsection{Solving Simple Additive TIVARY Model}

The general form of fuzzy goal programming is shown below:

Find X

$$
\begin{array}{ll}
\text { To satisfy } & \mathrm{f}_{\mathrm{i}}(\mathrm{x}) \\
\mathrm{AX} \leq \mathrm{g} & \\
\mathrm{X} \geq 0 &
\end{array}
$$

In which $\mathrm{f}(\mathrm{x})$ is fuzzy goal:

$\mathrm{Bi}$ is objective measure.

$\mathrm{Ax}<\mathrm{g}$ is systems limitation

$\mathrm{X}$ is $\mathrm{n}$-dimensional vector of decision variables

To solve the model, we use the concept of linear membership function. The membership function for the fuzzy goals, defined as follows:

If purpose $\mathrm{n}$ is in the form below, then: 
Find X

$$
\begin{array}{ll}
\text { To satisfy } & \mathrm{f}_{\mathrm{i}}(\mathrm{x}) \\
\mathrm{AX} \leq \mathrm{g} & \\
\mathrm{X} \geq 0 &
\end{array}
$$

Li is Low tolerance for fuzzy goal and bi-Li is tolerance interval. If fuzzy goal is $f_{i}(x) \stackrel{>}{\sim} b_{i}$, so membership function is as below:

$$
\mu_{i}=\left\{\begin{array}{cc}
1 & f_{i}(x) \leq b_{i} \\
\frac{u_{i}-f_{i}(x)}{u_{i}-b_{i}} & b_{i} \leq f_{i}(x) \leq u_{i} \\
0 & f_{i}(x) \geq u_{i}
\end{array}\right.
$$

In which ui is high tolerance and ui-bi is is tolerance interval.

Collective goal programming model becomes the sum of the membership functions of the objectives, which is formulated as follows:

$$
\begin{aligned}
& \operatorname{Max} V(\mu)=\sum_{i} \mu_{i} \\
& \text { Subject to } \\
& \mu_{\mathrm{i}}=\frac{f_{i}(x)-L_{i}}{b_{i}-L_{i}} \\
& A X \leq g \\
& \mu_{i} \leq 1 \\
& \mu_{i} \geq 0, X \geq 0
\end{aligned}
$$

We called $v(\mu)$ as Fuzzy availability function or fuzzy decision function. This is a single-objective linear programming problem that we can solve it using linear programming techniques, such as the simplex algorithm. Now, the two-objective problem is already described by this model. As we explained in the previous section, Beta problem is now as follows:

$$
\begin{aligned}
& \text { Max } 16500 y_{1}+22604 y_{2}+2851 y_{3} \sim 3658894 \\
& \text { Min } 1162 x_{1}+628 x_{2}+710 x_{3} \sim 2712728 \\
& \text { S.t } \\
& 0.5 x_{1}+0.25 x_{2}+0.25 x_{3}=y_{1} \\
& 0.25 x_{1}+0.15 x_{2}+0.60 x_{3}=y_{2} \\
& 0.60 x_{1}+0.40 x_{2}=y_{3} \\
& y_{1} \leq 16 \\
& y_{2} \leq 101 \\
& y_{3} \leq 390 \\
& x_{1}, x_{2}, x_{3}, y_{1}, y_{2}, y_{3} \geq 0 \\
& x_{1}, x_{2}, x_{3}, y_{1}, y_{2}, y_{3} \geq 0
\end{aligned}
$$


Upper bound for the purpose, income, is 3858894 and lower bound for the purpose, cost, is 2212728 , Now, we turn our fuzzy goals into membership functions.

$$
\begin{array}{r}
\mu_{1}=\frac{u_{1}-f_{1}(x)}{u_{1}-b_{1}}=\frac{3858894-\left(165000 y_{1}+226040 y_{2}+28513 y_{3}\right)}{3858894-3658894} \\
=\frac{3858894-\left(165000 y_{1}+226040 y_{2}+28513 y_{3}\right)}{200000} \\
\mu_{2}=\frac{f_{2}(x)-L_{2}}{b_{2}-L_{2}}=\frac{\left(11627 x_{1}+6288 x_{2}+7107 x_{3}\right)-2212728}{2712728-2212728} \\
=\frac{\left(11627 x_{1}+6288 x_{2}+7107 x_{3}\right)-2212728}{500000}
\end{array}
$$

Thus, it becomes a single-objective problem:

$$
\begin{aligned}
& \begin{array}{l}
\text { Max } V(\mu)=\mu_{1}+\mu_{2} \\
\text { Subject to }
\end{array} \\
& \mu_{1}=\frac{3858894-\left(165000 y_{1}+226040 y_{2}+28513 y_{3}\right)}{200000} \\
& \mu_{2}=\frac{\left(11627 x_{1}+6288 x_{2}+7107 x_{3}\right)-2212728}{500000} \\
& 0.5 x_{1}+0.25 x_{2}+0.25 x_{3}=y_{1} \\
& 0.25 x_{1}+0.15 x_{2}+0.60 x_{3}=y_{2} \\
& 0.60 x_{1}+0.40 x_{2}=y_{3} \\
& y_{1} \leq 16 \\
& y_{2} \leq 101 \\
& y_{3} \leq 390 \\
& \mu_{1} \leq 1 \quad, \mu_{2} \leq 1 \\
& x_{1}, x_{2}, x_{3}, y_{1}, y_{2}, y_{3}, \mu_{1}, \mu_{2} \geq 0
\end{aligned}
$$

If we solve this problem by using the software Lingo, We reach the optimum solution:

$$
x_{1}=200, \quad x_{2}=675, \quad x_{3}=2825, \quad y_{1}=16, \quad y_{2}=101, \quad y_{3}=390
$$

The amount of goals is equal to:

$$
\begin{aligned}
& b_{1}=16500 y_{1}+22604 y_{2}+2851 y_{3}=16500 \times 16+22604 \times 101+2851 \times 390=3658894 \\
& b_{2}=1162 x_{1}+628 x_{2}+710 x_{3}=1162 \times 200+628 \times 675+710 \times 2825=2662728
\end{aligned}
$$

Membership function values are as follows:

$$
\mu_{1}=1, \quad \mu_{2}=0.9
$$

4.4 Weighted Additive TIVARY Model:

Weighted additive models widely are used in goal programming and multi-objective programming techniques to reflect the relative importance of goals. In this approach, the decision maker can assign different weights to the coefficients of each term in the phase function. In objective function, each member will be multiplied by the appropriate factor, and then added together. Weighted additive model is as follows: 


$$
\operatorname{Max} V(\mu)=\sum_{i} w_{i} \mu_{i}
$$

Subject to

$$
\begin{aligned}
& \mu_{i}=\frac{f_{i}(x)-L_{i}}{b_{i}-L_{i}} \\
& A X \leq g \\
& \mu_{i} \leq 1 \\
& \mu_{i} \geq 0, X \geq 0
\end{aligned}
$$

$w_{\mathrm{i}}$ is relative importance from fuzzy goal (i). The main problem with this method of management is correct relative importance for fuzzy goals.

Now, we formulate and solve this problem by using weights $=\frac{1}{3}, \frac{2}{3}$. So the objective function is as follows:

$$
\operatorname{Max} V(\mu)=\frac{1}{3} \mu_{1}+\frac{2}{3} \mu_{2}
$$

Constraints of the problem will be the same with the simple mass. So we can solve it using the software Lingo and we arrive at the optimal solution:

$$
x_{1}=300, \quad x_{2}=525, \quad x_{3}=2654, \quad y_{1}=16, \quad y_{2}=101, \quad y_{3}=390
$$

The target value is equal to:

$$
b_{1}=3658894 \quad, \quad b_{2}=2562728
$$

Membership function values are as below:

$$
\mu_{1}=1, \quad \mu_{2}=0.7
$$

\section{Conclusions and Recommendations}

\subsection{Research Results}

According to research goals, in this study we follow 2 goals. These goals include reducing costs and increasing revenue for the production of products. The main way to achieve these goals was using the fuzzy goal programming. According to the research variables, TIVARY model was used through conventional methods. Finally, to achieve the objectives of the study, we used TIVARY simple additive model and TIVARY weighted mass model. First, we run the model using simple additive model.

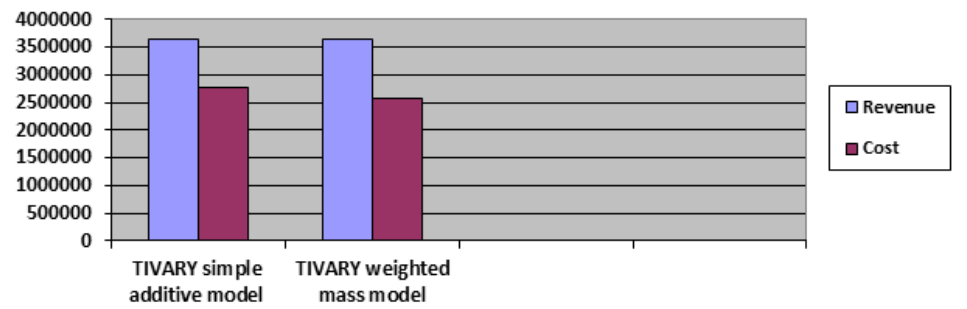

Figure 3. The results from two methods of TIVARY simple additive model and TIVARY weighted mass model

For the weighting of the criteria, we considered $1 / 3$ and $2 / 3$ for the cost and revenue, respectively. This weighting procedure was used, because the main objective is to reduce production costs. And we obtain lower cost with this weighting method. Figure 1 shows the results of the two models. 


\subsection{Research Proposing}

According to the results, the following suggestions are offered:

Determining the goal programming models is very important. And it is one of the most critical steps in developing models of goals. This is due to the different and changing views in this regard. It is suggested to consider the fuzzy goal programming model with fuzzy parameters. The amount of orders for goods is imported fuzzy model. In this case, it becomes clear that in which case it becomes clear that more reliable results can be generated in real terms.

Goal programming techniques can be used to solve other similar models in the organization. The degree of achievement, and variables, compared to be able to determine the most appropriate model.

\section{References}

Agawal, V., \& Kholi, P. V. (1991). Computer aided robot selection, the multiple attribute making approach. International Journal of Production Research, 29(8), 1629-1644.

Akoz, O., \& Petrovic, D. (2007). A fuzzy programming method with imprecise goal hierarchy. European Journal of Operational Research, 181(3), 1427-1433.

Anvarirostami, A., \& Tabatabai, A. (1998). Appraising the effectiveness of GP in incorporating the decision maker (DM) `s preferences. Journal of Operation Research Society of Japan, 41(2), 279-288.

Aouni, B., \& kettani, O, (1997). Martel, estimation through imprecise goal programming model. In Caballero, F.ruiz, \& R.E. Steuer (Eds), Advances in multiple objective and goal programming. Lecture Notes in Economics and Mathematical Systems, 455, 120-130.

Aouni, B., \& Kettani, O. (2001). Goal programming model: A glorious history and a promising future, European Journal of Operational Research, 133, 225-231.

Charnes, A., Cooper, W. W., \& Fergusen, R. (1995). Optimal estimation of executive compensation by linear programming. Management Science, 1, 138-151.

Chase, A. J. (2001). Operation management for completive advantage. Mc graw hill, ninth edition.

Chen, L. H., \& Tsai, F. C. (2001). Fuzzy goal programming with different importance and priorities. European Journal of Operational Research, 133(3), 548-556.

\section{Copyrights}

Copyright for this article is retained by the author(s), with first publication rights granted to the journal.

This is an open-access article distributed under the terms and conditions of the Creative Commons Attribution license (http://creativecommons.org/licenses/by/3.0/). 